Prevention and Rehabilitation

\title{
The effects of an 8-week knitting program on osteoarthritis symptoms in elderly women: A pilot randomized controlled trial
}

\author{
Guillaume Leonard ${ }^{\mathrm{a}, \mathrm{h},{ }^{*} \text {, Nicole Paquet }}{ }^{\mathrm{b}}$, Paulette Guitard ${ }^{\mathrm{c}}$, Karine Toupin-April ${ }^{\mathrm{c}, \mathrm{d}}$, \\ Sabrina Cavallo ${ }^{\mathrm{e}}$, Gail Paterson ${ }^{\mathrm{f}}$, Sibel Z. Aydin ${ }^{\mathrm{g}}$, Sibel Bakirci ${ }^{\mathrm{g}}$, \\ Alexia Coulombe-Lévêque ${ }^{\mathrm{a}, \mathrm{h}}$, Lucie Brosseau ${ }^{\mathrm{b}}$ \\ ${ }^{a}$ Faculté de médecine et des sciences de la santé, École de réadaptation, Université de Sherbrooke, Sherbrooke, Quebec, Canada \\ ${ }^{\mathrm{b}}$ Physiotherapy Program, School of Rehabilitation Sciences, Faculty of Health Sciences, University of Ottawa, Ottawa, Ontario, Canada \\ c Occupational Therapy Program, School of Rehabilitation Sciences, Faculty of Health Sciences, University of Ottawa, Ottawa, Ontario, Canada \\ d Children's Hospital of Eastern Ontario Research Institute, Department of Pediatrics, Faculty of Medicine, Ottawa, Ontario, Canada \\ e School of Rehabilitation Sciences, University of Ottawa, Ottawa, Ontario, Canada \\ ${ }^{\mathrm{f}}$ The Arthritis Society, Ottawa Office, Ontario Division, Ottawa, Ontario, Canada \\ ${ }^{g}$ Rheumatologist, Ottawa Hospital, Ottawa, Ontario, Canada \\ ${ }^{\mathrm{h}}$ Research Center on Aging, CIUSSS de l'Estrie - CHUS, Sherbrooke, Quebec, Canada
}

\section{A R T I C L E I N F O}

\section{Article history:}

Received 3 April 2020

Received in revised form

18 March 2021

Accepted 11 April 2021

\section{Keywords:}

Osteoarthritis

Hand

Exercise

Knitting

Women

Pain

Stiffness

\begin{abstract}
A B S T R A C T
Background: Exercise therapy is effective in reducing symptoms and disability associated with hand osteoarthritis (HOA) but often has low adherence. An intervention consisting in a meaningful occupation, such as knitting, may improve adherence to treatment. This pilot randomized controlled trial (RCT) studied the adherence and clinical effectiveness of a knitting program in older females suffering from HOA to evaluate the acceptability of this intervention and assess the feasibility of a larger-scale RCT. Methods: Single-blind, two-arm pilot RCT with a parallel group design with 37 participants (18 control, 19 intervention). Control participants were given an educational pamphlet and assigned to a waiting list. The knitting program (8-week duration) had two components: bi-weekly 20-min group knitting sessions and daily 20-min home knitting session on the 5 remaining weekdays. Measures included knitting adherence (implementation outcomes) as well as stiffness, pain, functional status, hand physical activity level, patient's global impression of change, health-related quality of life, self-efficacy, and grip strength (clinical outcomes measured throughout the 8-week program and 4 weeks after the intervention).

Results: Our protocol is feasible and the intervention was acceptable and enjoyable for participants, who showed high adherence. No difference was observed between the two groups for any of the clinical outcome measures (all $\mathrm{p}>.05$ ).

Conclusion: Knitting is a safe and accessible activity for older women with HOA. However, our 8-week knitting program did not result in improvements in any of our outcome measures. Knitting for a longer period and/or with higher frequency may yield better outcomes.
\end{abstract}

๑) 2021 Elsevier Ltd. All rights reserved.
Abbreviations: OA, Osteoarthritis; HOA, Hand Osteoarthritis; RCT, randomized controlled trial; QoL, quality of life; ACR, American College of Rheumatology; ID, identification number; PAR, Physical Activity Readiness; CONSORT, Consolidated Standards of Reporting Trials; ROM, range of motion; DIP, distal interphalangeal; PIP, proximal interphalangeal; CMC, carpometacarpal.

* Corresponding author. Research Center on Aging, CIUSSS de l'Estrie - CHUS, Canada.

E-mail addresses: guillaume.leonard2@usherbrooke.ca (G. Leonard), npaquet@ uottawa.ca (N. Paquet), guitardp@uottawa.ca (P. Guitard), ktoupina@uottawa.ca (K. Toupin-April), sabrina.cavallo@umontreal.ca (S. Cavallo), gpaterson@arthritis. ca (G. Paterson), Saydin@toh.ca (S.Z. Aydin), bakircisibel@gmail.com (S. Bakirci), alexia.coulombe-leveque@usherbrooke.ca (A. Coulombe-Lévêque), lbrossea@ uottawa.ca (L. Brosseau).

\section{Introduction}

Population aging, combined with rising healthcare costs and ever-limited healthcare resources, introduces growing challenges in the management of chronic diseases, including osteoarthritis (OA) (Walker and Helewa, 2004; Grotle et al., 2008; Denton \& Spencer, 2010; Liu-Ambrose et al., 2010). Women over 50 present a particularly high age-standardized total prevalence of hand osteoarthritis (HOA) compared to men of the same age (Cho et al., 2015). HOA is a debilitating condition resulting in pain, morning 
stiffness, finger joint inflammation and reduced grip strength (Cho et al., 2015), hindering participation in daily activities and threatening quality of life (QoL) (Liu et al., 2015; Kjeken et al., 2015).

Therapeutic exercises are a key component of the management of HOA (Davenport et al., 2012; Dziedzic et al., 2015; Hennig et al., 2015; Brosseau et al., 2018). Exercise programs that include range of motion (ROM), stretching, or strengthening exercises have been shown to effectively reduce pain (Davenport et al., 2012; Hennig et al., 2015; Lefler and Armstrong, 2004; Østerås et al., 2013; Villafañe Cleland \& Fernandez-De-Las-Penas, 2013; Merritt, 2012); reduce joint stiffness (Hennig et al., 2015; Østerås et al., 2013; Merritt, 2012); decrease fatigue (Hennig et al., 2015); improve physical function (Davenport et al., 2012; Hennig et al., 2015; Østerås et al., 2013; Dziedzic et al., 2015; Merritt, 2012); and improve grip strength (Hennig et al., 2015). Functional exercises (e.g., writing, holding plates, opening clothes pegs, tearing sheets of paper, fastening buttons) have also been proven effective in the management of HOA, with positive effects noted on pain, physical function, and pinch strength (Davenport et al., 2012). Unfortunately, while the benefits associated with these exercise programs have been demonstrated, dropout rates range from $0 \%$ to $53 \%$ (Davenport et al., 2012; Hennig et al., 2015; Østerås et al., 2013; Dziedzic et al., 2015; Villafañe et al., 2013; Merritt, 2012) and exercise adherence (measured in only three studies) range from $97 \%$ to 47\% (Lefler and Armstrong, 2004; Østerås et al., 2013; Dziedzic et al., 2015).

According to the Canadian Model of Occupational Performance and Engagement (CMOP-E), knitting can be a purposeful and meaningful occupation (Polatajko et al., 2007): Knitting may be more appealing, motivating, enjoyable and rewarding than traditional therapeutic exercises (Krauss et al., 2017). This may in turn promote adherence to the proposed activity, especially in elderly women (Schutzer and Graves, 2004). Knitting also represents a promising treatment approach for older individuals suffering from HOA because of its potential effect on physiological, psychological, and social factors (Hoeger Bement et al., 2009; Koltyn and Umeda, 2007; Hoffman, 2008; Damush et al., 2005).

The objective of this pilot RCT was to collect data on the adherence to an 8-week knitting program in older females suffering from HOA, as well as preliminary data on the immediate and short-term (4-week post-intervention) effect of such a program on morning stiffness, pain, functional status, hand and physical activity level, patient's global impression of change, health-related QoL, self-efficacy and grip strength.

\section{Methods}

The methodology of this pilot randomized controlled trial (RCT) is in line with the CONSORT statement. The pilot study ran from August to December of 2017 (4-month duration).

\subsection{Study design}

The study was a two-arm pilot RCT with a parallel group design comparing two study groups: Participants in group 1 (intervention group) received the knitting intervention and were given an educational pamphlet on OA designed by The Arthritis Society (TAS); participants in group 2 (control group) received only the educational pamphlet (TAS, 2009) and were assigned to a waiting list. Immediate effects were measured throughout the 8-week knitting program, and retention effects were measured 4 weeks post-intervention (follow-up). Given the nature of the intervention, neither the participants nor the therapists/knitting instructors were blinded. However, the trained independent evaluator (VB, a kinesiologist) conducting the performance evaluations and calculating the scores of the self-reported questionnaires was blinded. These assessments were conducted four times: Baseline, midintervention (week 4), immediately post-intervention (week 8), and four weeks post-intervention (week 12). The study protocol was approved by the ethics committee of the Research Ethics Boards of the University of Ottawa (\#H02-16-12).

\subsection{Study population}

To be eligible for this pilot RCT, participants were required to: 1) meet the clinical and radiographic criteria of definite HOA set out by the American College of Rheumatology (ACR) (Altman et al., 1990) and have experienced pain symptoms for at least 3 months; 2) be a woman aged between 50 and 85 years; 3 ) have moderate to severe morning stiffness (defined as a score $\geq 4$ on a $10 \mathrm{~cm}$ visual analogue scale $[0=$ no stiffness; $10 \mathrm{~cm}=$ worst imaginable stiffness], (Vlieland et al., 1997) at the time of study entry; 4) display X-ray evidence of joint space narrowing of the hands (Kallman et al., 1989); 5) have no previous experience with knitting or have not knitted in the last 6 months; 6 ) be available for sessions at the seniors' club twice weekly; and 7) be able to understand written and verbal English instructions. Participants were excluded if they: 1) suffered from other orthopedic or rheumatologic diseases (e.g., inflammatory arthritis), or showed evidence of chondrocalcinosis; 2) had a history of finger joint surgery; 3) suffered from an acute disease, such as uncontrolled diabetes mellitus, untreated hypertension, neurological deficits (motor or sensory), cognitive deficit or mental health conditions; 4) were taking OA medication that was expected to change during the study period; 5) were receiving current rehabilitation treatments or any other painrelated treatment besides medication for $O A ; 6$ ) had received a corticosteroid injection in a finger joint within the last 6 months; or 7) planned to move outside the region within 6 months.

A rheumatologist (SB) made the HOA diagnosis, in accordance with the ACR clinical criteria (Altman et al., 1990) and the radiologic criteria of Kallman et al. (1989). Recent ( $<1$ year) hand X-ray reports (interpreted by a radiologist) were also reviewed by two rheumatologists (SZA, SB) to confirm diagnosis. The ACR classification was used to assess the presence of (1) pain, aching, or joint stiffness; (2) bony enlargement of 2 or more of 10 selected finger joints; (3) bony enlargement of at least one distal interphalangeal (DIP) joint; and (4) fewer than 2 swollen metacarpophalangeal (MCP) joints, or (5) deformity of at least 1 of 10 selected joints (including the CMC joint, the thumb interphalangeal joint, the 4 PIP joints, and the 4 DIP joints).

\subsection{Recruitment}

Participants were recruited via advertisements placed in local newspapers, information letters sent to local rheumatologists, and posters placed at the TAS office/Facebook page and in the waiting rooms of each rheumatologist's office. Potential participants were invited to complete an online eligibility and admission questionnaire (Brosseau et al., 2014), including socio-demographic and handedness information (Oldfield, 1971; Veale, 2014), as well as clinical information such as location, intensity, timing and duration of morning stiffness and pain (Orbai et al., 2015; Vlieland et al., 1997; Scott et Huskisson, 1976). If they passed this first screening process, participants were invited to meet with the research coordinator to confirm eligibility, and then to give their informed consent. 


\subsection{Group assignment}

Eligible participants were assigned to a group (ratio 1:1) by way of central randomization (Van Tulder et al., 1997). The randomization schedule was prepared by the study's biostatistician using a sequence of computer-generated random numbers (using statistical software SAS macro in SAS 9.3) and a blocking factor (randomly varying between 4 and 6). Participants in group 2 (control group) were assured access to the knitting program after completion of the pilot RCT.

Numbered and sealed opaque envelopes were prepared by an independent staff member and opened in sequence by each participant after they had signed the consent form and completed all baseline measurements. These envelopes contained the information regarding group allocation (knitting vs. control group). Once they had opened their envelopes, the participants met with the research assistant, who provided assignment-specific instructions for the next steps. The independent outcome evaluator was blinded to group allocation, and participants in both groups were requested not to disclose details regarding their group allocation to the independent outcome evaluator at any time. Before running the randomization program, the data manager documented the participants' initials and date of birth (month and year). After running the program, the data manager documented the intervention assignment for each participant, assigned them a study identification (ID), and informed the research assistant of the participants' ID and intervention assignment. This process ensured that allocation was concealed. Participants were then informed of their group allocation.

\subsection{Intervention}

Participants in both groups were given an educational pamphlet on HOA. No instructions were given to participants regarding how often they should read the educational pamphlet. All participants received their regular medical care during the study period but were asked to refrain from using pain-reducing modalities such as ointments and cryo- and thermotherapy; to avoid hand exercises (other than knitting) and massages; and not to wear hand or finger splints for the duration of the experiment. As this was not always possible for every participant, we asked participants to complete a daily activity log to keep track of these possible confounding factors.

Intervention group. The knitting program is reported based on the 16 criteria of the Consensus on exercise reporting template (CERT), a tool that guides the reporting of essential components of exercise programs (Slade et al., 2016). The intervention included two types of knitting sessions: 1 ) bi-weekly knitting group sessions held at a seniors' club in metropolitan Ottawa, beginning early morning at 8:00 a.m. in the presence of other regular knitters/members; and 2) daily individual sessions, held at home (unsupervised) and performed in the hour after waking up in the morning. Both types of sessions lasted $20 \mathrm{~min}$. The group sessions took place on Tuesday and Thursday mornings, and the home sessions took place every remaining morning, for a total of 56 sessions ( 7 sessions per week for 8 weeks). Study participants joined the regular senior's club members in knitting wool blankets for sick children and older individuals, using the English knitting style (see Fig. 1). The blankets were made of individual squares pieced together. Participants were encouraged to knit one wool square per week and to perform similar knitting sessions at home. Participants used size 5, 6 or 7 (US size, corresponds to 7, 8, 9 UK imperial size) knitting needles and \#4 yarn (Standard Yarn Weight System; http://www.craftyarncouncil.com/ weight.html). The knitting instructors were two members of the research team [NP \& PG] and two members of the Pacesetters
Seniors Centre. One of the instructors of the research team (who was a physiotherapist) had knitted for more than 12 years. The other instructor (occupational therapist, with 45 years of experience in knitting) also had clinical experience in helping and teaching patients to knit after episodes of long-term disability. All instructors ensured that participants followed the prescribed program, and that they properly filled out the individual logbooks in which they recorded their daily stiffness, pain levels, and hand activities. Participants who failed to attend 2 consecutive group knitting sessions were contacted by phone by the research assistant to encourage them to attend the next session. At the end of the 8-week intervention, participants were asked to refrain from knitting until the follow-up assessment ( 4 weeks later), so that we could measure the retention effect of the knitting program.

Control group. Participants assigned to the control group were placed on a waiting list for the duration of the knitting program and the 4-week follow-up period, for a total of 12 weeks. They were encouraged to read the educational pamphlet (same pamphlet as participants in the intervention group), and to carry on with their usual routine. Control participants were not permitted to attend knitting sessions at the senior's club during the study period. Once the study was completed, the knitting sessions were made available to all study participants.

\subsection{Outcome measures}

\subsubsection{Implementation outcomes}

Adherence to the knitting program was estimated using the logbook and the seniors club attendance sheet, whereby we divided the number of knitting sessions completed (at the club and at home) by the number of knitting sessions prescribed (56 sessions).

Dropout rates were recorded in both groups and blinding of the outcome evaluator was assessed through a questionnaire used in a previous RCT on HOA (Brosseau et al., 2005), wherein the evaluator was asked, after the intervention (week 8), to identify which group each participant belonged to.

The number of wool squares knitted and information about knitting technique were recorded in participants' logbooks, as well as adverse events and changes in habits, physical activity, and medication intake, so that we could monitor the frequency of occurrence of these possible confounding factors. Knitting enjoyment was also assessed using the binary question "Do you enjoy knitting?"

\subsubsection{Clinical outcomes}

The clinical outcome measures were selected according to the OMERACT framework (Kloppenburg et al., 2015) and included the following:

Stiffness and pain levels were recorded using the $0-100$ visual analog scale provided in the logbook (daily measures) and in the questionnaires (periodic measures obtained at baseline, midintervention, immediately post-intervention, and 4 weeks postintervention reflecting general pain at rest, general pain during activity, and general morning stiffness). For daily measures (assessed from week 1 to week 12), participants rated the severity of their symptoms 5 times daily: When waking up in the morning; immediately post-knitting (intervention group) or 1-h postawakening (control group); 2- and 4-h post-awakening; and in the evening. To simplify data presentation and analyses, week average values were calculated for each of these five time points. Daily stiffness $2 \mathrm{~h}$ post-awakening was the primary outcome measure.

Functional status was assessed using the Australian/Canadian Osteoarthritis Hand Index (AUSCAN), a validated, reliable, and 

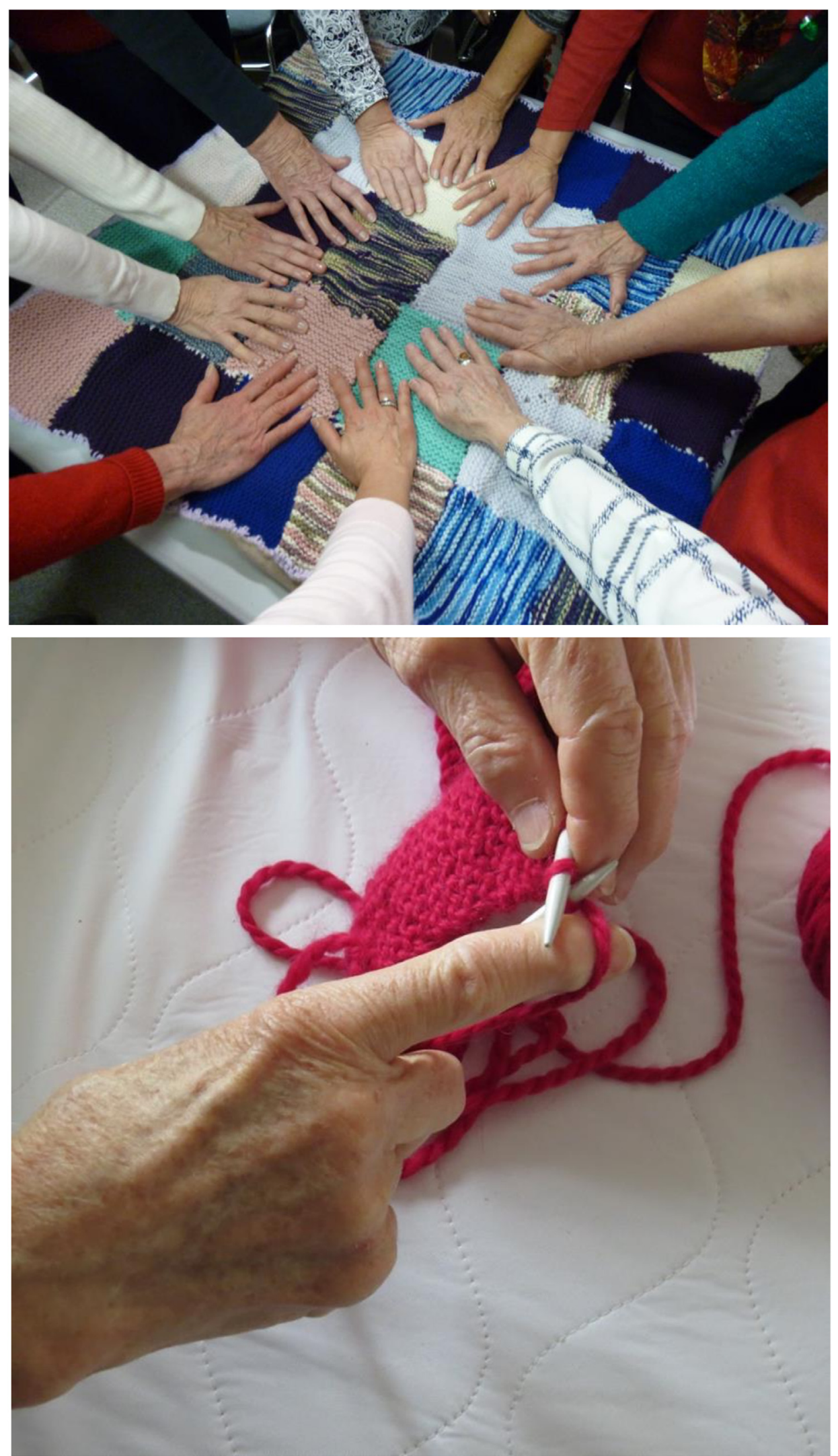

Fig. 1. Knitting intervention. 
responsive measurement scale for HOA (Bellamy et al., 1996, 2002a, 2002b; Allen et al., 2007). The AUSCAN consists in a 15-item scale, with items grouped into three subsections: A) pain intensity, B) stiffness severity, and C) hand functional status/difficulty in activities of daily living. For each item, scores range from 0 (no symptoms) to 4 (severe symptoms).

Hand physical activity was measured using an adapted version of the 7-Day Physical Activity Readiness (PAR) (Sallis et al., 1985, 1997; Hayden-Wade et al., 2003). This validated instrument is in the form of a calendar in which participants record the duration (minutes per day) and frequency (days per week) of physical activity. For this study, participants' logbooks included an adapted version of the 7-day PAR in which physical activity (including knitting for the intervention group) was recorded.

Patient's global impression of change was assessed by asking patients if, following the knitting program, their condition was: 1) very much improved; 2 ) much improved; 3 ) minimally improved; 4) no change; 5) minimally worse; 6) much worse; 7) very much worse (Guy, 1976).

Health-related QoL was evaluated using the EuroQoL Index (EQ5D-5L) (Rabin and Charro, 2001), which covers mobility, self-care, daily activities, pain/discomfort, and anxiety/depression subscales.

Self-efficacy was assessed using The Stanford Arthritis SelfEfficacy Scale (Lorig et al., 1989), consisting in statements which patients rate on a ten-point scale ranging from 1 (very uncertain) to 10 (very certain).

Bilateral hand grip strength was measured by the independent outcome evaluator using a portable JAMAR Hydraulic Hand Dynamometer in the standardized sitting position (Sammons Preston Rolyan, Bolingbrook, IL, USA). This is a highly reliable outcome measure in older individuals and in persons with hand conditions/ injuries (Villafane et al., 2015; Bohannon, 1997; Schreuders et al., 2003).

\subsection{Measurement frequency, methods, and procedures}

Stiffness and pain were measured multiple times daily, whereas implementation and other clinical outcomes were evaluated four times: At baseline, mid-intervention (week 4), post-intervention (week 8) and at follow-up (week 12). Independent evaluation sheets were used for each patient for each of these four assessment sessions to minimize recording bias. All self-reported questionnaires were filled out on a laptop using Survey Monkey®. These evaluations took approximately $60 \mathrm{~min}$ to complete in a private assessment room to optimize blinding of the outcome evaluator and to reduce record bias (information bias).

\subsection{Statistical analysis}

Descriptive statistics were used to summarize variables, to assess the distributional assumptions of the statistical techniques used, and to depict implementation outcomes. Visual inspection of the histograms and Shapiro-Wilk tests suggested that the data were normally distributed, and parametric tests were used. Baseline characteristics of participants were compared using independent sample t-tests, and clinical outcomes were compared using a 2-way repeated analysis of variance (ANOVA) with the between factor Group (knitting vs. control) and within factor Time (baseline, 4week, 8-week, and 4-week follow-up). The significance level was set at 0.05 for all analyses. No interim efficacy or subgroup analyses were planned.

\section{Results}

\subsection{Study sample}

One hundred and thirteen (113) individuals were screened for eligibility. From these 113 individuals, 62 were excluded after completing the online eligibility and admission questionnaire, for not meeting the inclusion criteria (see Fig. 2). These individuals were excluded from participating because they had undergone surgery of one or more finger joints $(n=5)$; had received a cortisone injection in the last 3 months $(n=5)$; were younger than 50 years old $(n=1)$; displayed impaired hand function due to other health conditions $(\mathrm{n}=24)$; had an absence of morning symptoms $(\mathrm{n}=18)$; were actively involved in knitting $(\mathrm{n}=8)$; or had language limitations (unable to understand written and verbal English instructions $(\mathrm{n}=1)$.

The research coordinator was able to confirm the eligibility of all remaining potential participants, but 14 additional individuals declined to participate for the following reasons: Not available twice a week $(\mathrm{n}=10)$; no interest in knitting $(\mathrm{n}=1)$; holiday plans $(\mathrm{n}=1)$; living too far $(\mathrm{n}=1)$; and family member illness $(\mathrm{n}=1)$. The remaining $37(\mathrm{n}=37)$ participants were randomly assigned to the intervention (knitting; $n=19$ ) or control (waitlist; $n=18$ ) group. After the baseline assessment, two $(n=2)$ knitters and one $(\mathrm{n}=1)$ control subject were lost, and after the 4-week assessment, another two $(\mathrm{n}=2)$ knitters and two $(\mathrm{n}=2)$ controls quit; thus 15 $(n=15)$ participants remained in each group (see Fig. 2 ).

\subsection{Characteristics of the participants}

No statistically significant differences were observed between groups at baseline, except for age $(\mathrm{p}=0.01)$ and left-hand grip strength $(\mathrm{p}=0.04$; see Table 1$)$.

\subsection{Implementation outcomes}

Dropout rates. The overall dropout rate was $18.2 \%$, with a $21.1 \%$ dropout rate in the knitting group ( $\mathrm{n}=4$ dropouts, from an initial $\mathrm{n}=19$ ) and a $16.7 \%$ dropout rate in the control group ( $\mathrm{n}=3$ dropouts, from an initial $n=18$ ). Reasons for dropout are detailed in Fig. 2 and include not having preferred group allocation $(\mathrm{n}=1)$; hand injuries (unrelated to the knitting intervention; $n=2$ ); unavailability (unable to attend knitting sessions; $n=1$ ); loss of interest $(n=2)$; and illness of a relative $(n=1)$. The 2 participants who lost interest in the project had been randomized to the control group. In the knitting group, hand injuries (not related to the knitting intervention) were the most common reason for dropout. Only two participants in the knitting group discontinued participation for reasons related to the intervention (group allocation and unavailability). There were no losses to follow-up in either the knitting group or control group.

Attendance to knitting sessions. On average, every week participants attended $75 \%$ of home + group sessions ( 5.25 out of 7 ; see Table 2) and $80 \%$ of group sessions (1.6 out of 2 ).

Blinding. The outcome evaluator was able to correctly identify the group to which participants belonged in $16 \%$ of the cases, which suggests that blinding was successful.

Adverse events. No participant reported adverse events during or after the knitting intervention. Dropouts were not related to the knitting activity, but rather caused by a lack of motivation to participate in the study, hand or wrist injuries resulting from a fall, and because of severe illness afflicting a family member. 


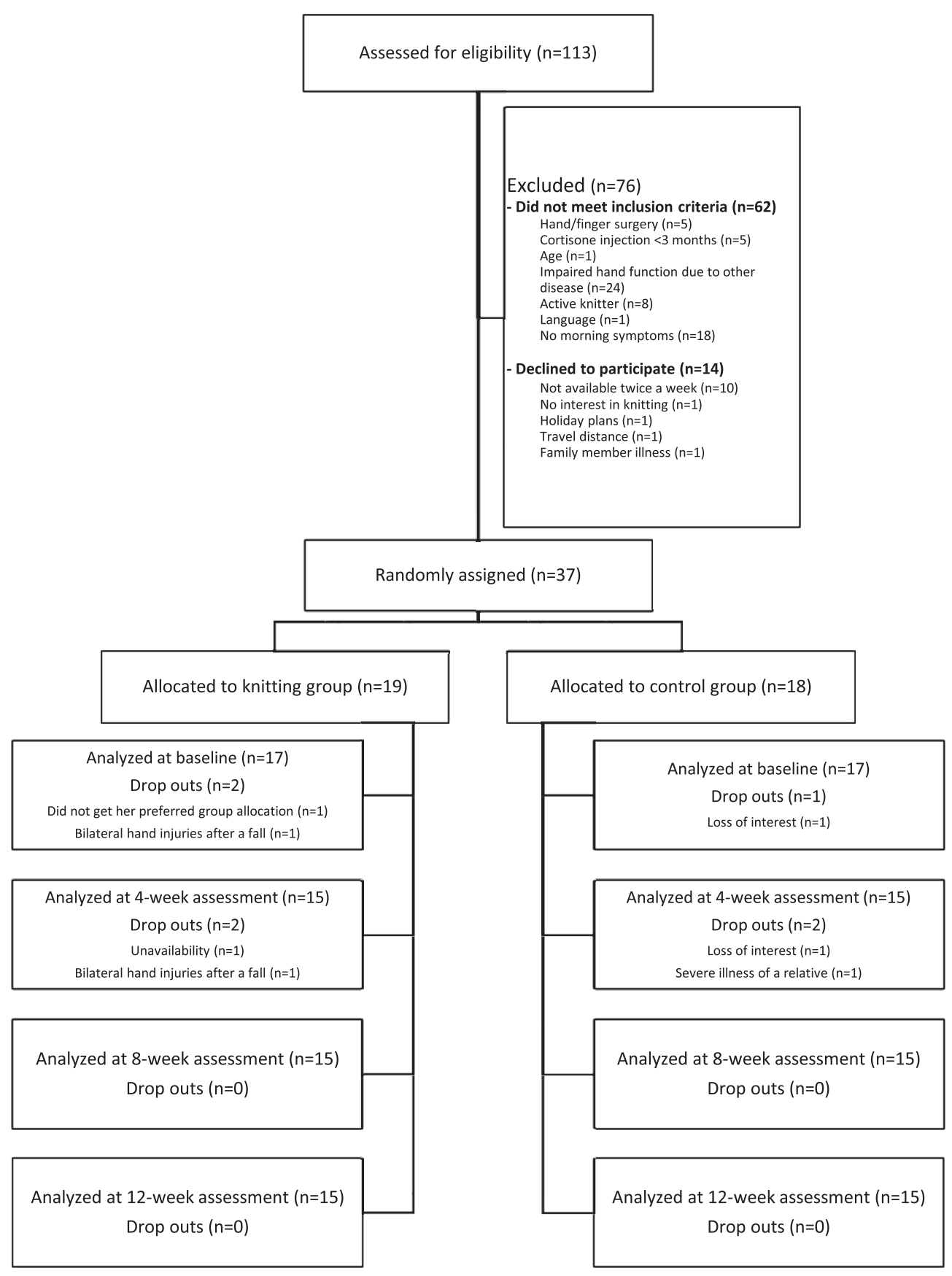

Fig. 2. Study flow diagram.

Knitting enjoyment. All remaining participants in our intervention group $(n=15)$ reported (in their daily $\log$ ) that they enjoyed knitting throughout the first 6 weeks of the experiment; this number decreased slightly during weeks $7-8$, with $92.9 \%$ of participants reporting that they enjoyed knitting.

\subsection{Clinical outcomes}

\subsubsection{Daily pain and stiffness and pain}

Throughout the study, participants recorded their levels of stiffness and pain five times daily: When waking up in the morning; immediately post-knitting (intervention group) or 1 -h postawakening (control group); 2 - and 4-h post-awakening; and in the evening. As can be seen from Fig. 3 (depicting stiffness and pain 2-h awakening), there was no time or group-effect for these measures. Visual inspection and statistical analyses (not shown) confirmed the same pattern of results for the other time measures of daily stifness and pain (all p-values $>.05$ ).

\subsubsection{Secondary clinical outcomes}

There was a statistically significant time effect for morning pain during activity $\left(\eta_{\mathrm{p}}^{2}=0.15, \mathrm{p}=.003\right)$ and for minutes spent doing hand physical activity $\left(\eta_{\mathrm{p}}^{2}=0.12, \mathrm{p}=.047\right)$, indicating that both groups improved over time. However, there was no significant between-group difference (all p-values $>.05$ ) for these measures and any other secondary clinical outcomes (periodic measure of stiffness and pain, functional status, hand and physical activity level, patient's global impression of change, health-related QoL, 
Table 1

Characteristics of participants.

\begin{tabular}{|c|c|c|c|c|}
\hline & All patients $(\mathrm{n}=37)$ & Knitting group $(\mathrm{n}=19)$ & Control group $(n=18)$ & p-value* \\
\hline Age (years) & $67.5 \pm 7.2$ & $64.6 \pm 6.4$ & $70.5 \pm 7.0$ & 0.01 \\
\hline Level of education & & & & 0.63 \\
\hline Lower secondary school & $2(5.4)$ & $0(0)$ & $2(11.1)$ & \\
\hline Upper secondary school & $5(13.5)$ & $3(15.8)$ & $2(11.1)$ & \\
\hline College & $8(21.6)$ & $5(26.3)$ & $3(16.7)$ & \\
\hline University & $22(59.5)$ & $11(57.9)$ & $11(61.1)$ & \\
\hline Living alone & $5(16.7)$ & $1(6.7)$ & $4(26.6)$ & 0.33 \\
\hline Retired & $34(91.9)$ & $17(89.5)$ & $17(94.4)$ & 1 \\
\hline Dominant hand & & & & 1 \\
\hline Right hand dominant & $33(89.2)$ & $17(89.5)$ & $16(88.9)$ & \\
\hline Left hand dominant & $3(8.1)$ & $2(10.5)$ & $1(5.6)$ & \\
\hline Ambidextrous & $1(2.7)$ & $0(0)$ & $1(5.6)$ & \\
\hline Disease duration (years) & $10.5 \pm 7.5$ & $9.7 \pm 5.5$ & $11.3 \pm 9.2$ & 0.51 \\
\hline Level of HOA & & & & 1 \\
\hline Mild & $14(37.8)$ & $7(36.8)$ & $7(38.9)$ & \\
\hline Moderate & $19(51.4)$ & $10(52.6)$ & $9(50.0)$ & \\
\hline Severe & $4(10.8)$ & $2(10.5)$ & $2(11.1)$ & \\
\hline Both hands affected & $37(100)$ & $19(100)$ & $18(100)$ & - \\
\hline General pain at rest $(0-100)$ & $26.8 \pm 19.4$ & $26.3 \pm 18.0$ & $27.2 \pm 21.4$ & 0.89 \\
\hline General pain during activity $(0-100)$ & $40.5 \pm 16.5$ & $43.2 \pm 15.3$ & $37.8 \pm 17.7$ & 0.33 \\
\hline General morning stiffness $(0-100)$ & $42.7 \pm 16.4$ & $43.2 \pm 12.9$ & $42.2 \pm 19.9$ & 0.87 \\
\hline AUSCAN - pain $(0-20)$ & $10.5 \pm 3.1$ & $10.7 \pm 3.2$ & $10.2 \pm 3.1$ & 0.66 \\
\hline AUSCAN - stiffness $(0-4)$ & $2.3 \pm 0.9$ & $2.4 \pm 0.9$ & $2.2 \pm 1.0$ & 0.52 \\
\hline AUSCAN - function $(0-36)$ & $20.1 \pm 5.8$ & $19.6 \pm 6.9$ & $20.7 \pm 4.5$ & 0.57 \\
\hline EQ-5D - Quality of life $(0-20)$ & $4.5 \pm 2.3$ & $4.6 \pm 2.4$ & $4.4 \pm 2.4$ & 0.76 \\
\hline Arthritis self-efficacy pain subscale $(1-10)$ & $7.4 \pm 1.6$ & $7.4 \pm 1.6$ & $7.4 \pm 1.6$ & 0.90 \\
\hline Presence of hand pain, aching or stiffness & $36(100)$ & $18(100)$ & $18(100)$ & - \\
\hline \multicolumn{5}{|c|}{ No. of joints with hard tissue enlargement $(0-10)$} \\
\hline Right hand & $5.8 \pm 2.4$ & $5.8 \pm 2.1$ & $5.7 \pm 2.6$ & 0.80 \\
\hline Left hand & $5.3 \pm 2.5$ & $5.4 \pm 2.0$ & $5.2 \pm 2.9$ & 0.83 \\
\hline \multicolumn{5}{|l|}{ No. of swollen joints $(0-5)$} \\
\hline Right hand & $0.2 \pm 0.6$ & $0.1 \pm 0.2$ & $0.4 \pm 0.8$ & 0.19 \\
\hline Left hand & $0.1 \pm 0.5$ & $0 \pm 0$ & $0.2 \pm 0.7$ & 0.32 \\
\hline \multicolumn{5}{|c|}{ No. of joints with hard tissue enlargement of DIP joints $(0-5)$} \\
\hline Right hand & $3.7 \pm 1.2$ & $3.8 \pm 1.1$ & $3.7 \pm 1.3$ & 0.79 \\
\hline Left hand & $3.4 \pm 1.4)$ & $3.4 \pm 1.2)$ & $3.4 \pm 1.7$ & 0.91 \\
\hline \multicolumn{5}{|l|}{ No. Of joints with deformity $(0-10)$} \\
\hline Right hand & $0.8 \pm 1.3$ & $0.7 \pm 1.1$ & $0.9 \pm 1.4$ & 0.52 \\
\hline Left hand & $0.8 \pm 1.3$ & $0.6 \pm 1.1$ & $0.9 \pm 1.5$ & 0.38 \\
\hline Presence of tenderness & $2 \pm 5.6$ & $1 \pm 5.6$ & $1 \pm 5.6$ & 1 \\
\hline \multicolumn{5}{|l|}{ Mean of grip strength (kg) } \\
\hline Right hand & $13.4 \pm 5.4$ & $13.9 \pm 5.3$ & $12.9 \pm 5.7$ & 0.59 \\
\hline Left hand & $12.3 \pm 5.9$ & $14.1 \pm 4.6$ & $10.3 \pm 6.5$ & 0.04 \\
\hline \multicolumn{5}{|l|}{ Medication } \\
\hline Analgesics (yes) & $20(55.6)$ & $8(44.4)$ & $12(66.7)$ & 0.32 \\
\hline NSAIDs (yes) & $13(36.1)$ & $5(27.7)$ & $8(44.0)$ & 0.49 \\
\hline Glucosamine (yes) & $3(8.3)$ & $1(5.6)$ & $2(11.1)$ & 1 \\
\hline
\end{tabular}

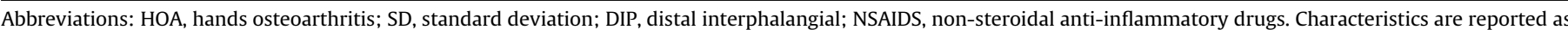
number (proportion) or mean \pm SD.

Table 2

Number of days of knitting at home and at the club.

\begin{tabular}{|c|c|c|c|c|c|c|c|c|}
\hline & Week 1 & Week 2 & Week 3 & Week 4 & Week 5 & Week 6 & Week 7 & Week 8 \\
\hline & $\mathrm{n}=17$ & $\mathrm{n}=17$ & $\mathrm{n}=17$ & $\mathrm{n}=15$ & $\mathrm{n}=15$ & $\mathrm{n}=15$ & $\mathrm{n}=15$ & $\mathrm{n}=15$ \\
\hline Mean & 6.24 & 6.06 & 5.76 & 5.59 & 5.71 & 6.06 & 5.88 & 5.88 \\
\hline SD & 1.821 & 2.304 & 2.278 & 2.238 & 2.285 & 2.304 & 2.288 & 2.261 \\
\hline
\end{tabular}

self-efficacy, and grip strength) at baseline, mid-intervention (week 4), post-intervention (week 8) and follow-up (week 12).

\section{Discussion}

The data collected in this pilot RCT demonstrate that our protocol, including the recruitment of older women with HOA and our data collection procedure, is feasible, and that the 8-week knitting program proposed as the intervention can be well tolerated.
Recruitment was completed in the planned time frame (4 months) and there were no missing data. Both adherence to the knitting program and knitting enjoyment were relatively high amongst participants. In past RCTs on traditional therapeutic exercise for HOA (strength training, range-of motion and stretching; Lefler and Armstrong, 2004; Østerås et al., 2013; Dziedzic et al., 2015), exercise adherence ranged from $47 \%$ to $94 \%$. The knitting program in this pilot RCT had a 75\% adherence rate. Many factors could have positively influenced the adherence rate to knitting, 


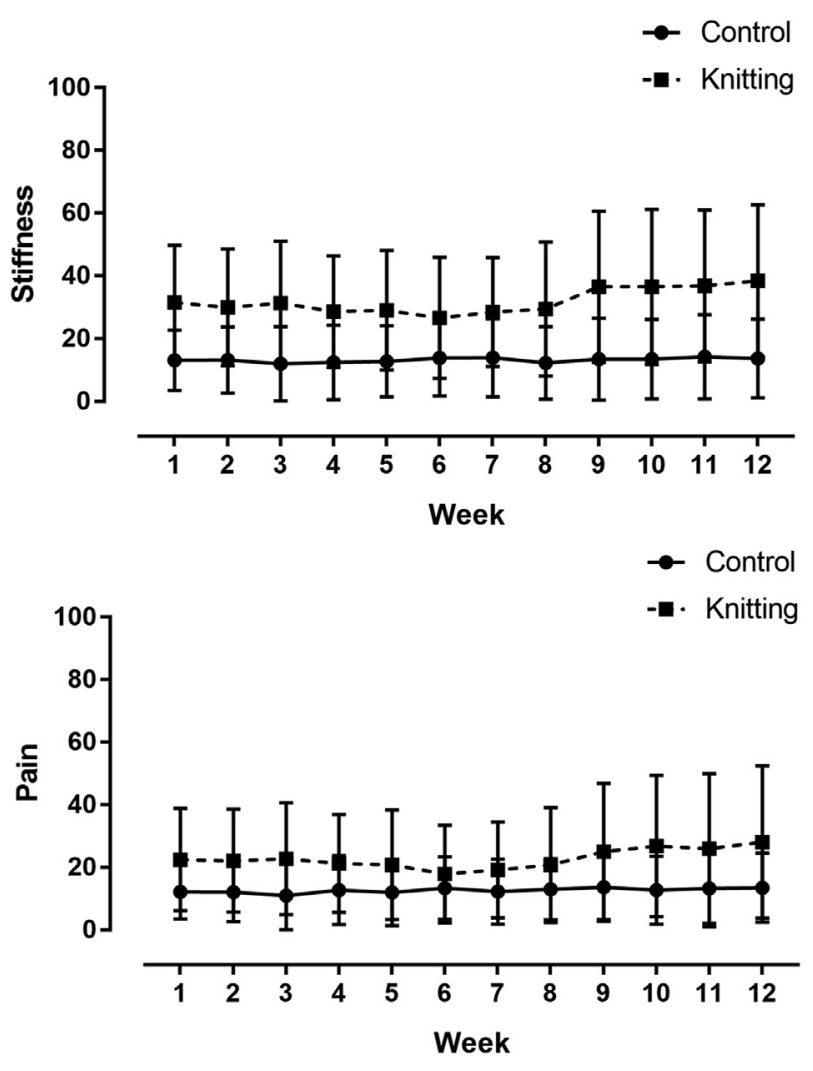

Fig. 3. Daily stiffness and pain $2 \mathrm{~h}$ post-awakening. Week average values (mean $\pm \mathrm{SD}$ ) representing daily stiffness and pain levels measured 2-h post-awakening. There was no significant difference between the two groups for every time points, and no significant time effect (all p-values $>.05$ ), suggesting that knitting did not affect daily stiffness and pain.

including the close follow-up monitoring during the study period especially during the group sessions at the seniors' club (Hennig et al., 2015; O'Brien, 2012). Moreover, knitting is generally a meaningful activity that people choose to partake in, rather than a prescribed exercise program in which they may have no interest (Mee \& Sumsion 2001; Polatajko et al., 2007; Roberts \& Banningan, 2018). Exchanging with other seniors at the club and making blankets to benefit sick children and elderly patients might have further contributed to increase motivation. Comparisons with other studies for exercise adherence nevertheless remains hazardous, given the differences in relation to the duration of the intervention (6 weeks for Lefler \& Armstrong [2004] vs 12 weeks for Østerås et al., [2013] and Dziedzic et al., [2015]), frequency of the exercises (once a week for Dziedzic et al., [2015] vs 3 times weekly for Østerås et al., [2013]), duration of the follow-up (up to 12-month for Lefler \& Armstrong [2004]), as well as the metrics used to evaluate adherence (see for instance Østerås et al., [2013]).

Exercise programs are known to have beneficial effects on pain, mood, and social interactions (Bement et al., 2009a; Hoffman and Hoffman 2008; Wampold 2001; Bement et al., 2009b; Shankland et al., 2017; Brosseau and Léonard, 2017). It stood to reason that a program involving knitting (a functional and meaningful activity), particularly in the setting of a seniors' club, could promote these effects in women suffering from HOA. The potential benefits of knitting for HOA were supported by a recent case study, which found that knitting (daily sessions over 12 weeks, > 20 min) significantly reduced morning stiffness and pain in an 86-year-old woman diagnosed with moderate to severe bilateral HOA (Brosseau and Leonard, 2017). However, results from this pilot study showed that there was no clinically or statistically significant difference between the two groups for any of the outcome measures, suggesting that the knitting intervention did not help reduce HOA symptoms in our participants. Yet, at this point, we must be careful and avoid any conclusions regarding the clinical effectiveness, as this pilot study included only a small number of participants, which increases the probability of committing type-II errors. Still, our study offers valuable insights for the design of future and adequately powered studies looking at clinical effectiveness. For example, although there were no between-group differences, we observed statistically significant reduction in general pain during activity and an increase in time spent doing hand physical activity. Future studies should probably look at the effect of knitting on these outcomes, rather than on daily morning stiffness.

These results are not consistent with past studies showing that exercise can reduce pain in healthy individuals and in patients suffering from chronic pain (Ye et al., 2011; Bertozzi et al., 2015; Aebischer et al., 2016; Valdes and Marik 2010). Indeed, several other studies have demonstrated that resistance exercises can result in antinociception (Koltyn and Arbogast 1998; Galdino et al., 2014). Training programs consisting in stretching, ROM, isotonic and isometric contractions (which incidentally are all involved in knitting) have also been shown to effectively reduce pain (Davenport et al., 2012; Hennig et al., 2015; Lefler and Armstrong, 2004; Østerås et al., 2013; Villafañe et al., 2013; Merritt, 2012; Bement et al., 2009a; Bement et al., 2009b), presumably via the release of endogenous opioids and growth factors, and the activation of nociceptive inhibitory mechanisms (Ray and Carter, 2007; Millan, 2002). Longer duration of activity (i.e., $30 \mathrm{~min}$ ) appears to be necessary to trigger exercise-induced hypoalgesia in healthy individuals (Bement, 2009b; Koltyn, 2002), and the duration and consistency of therapeutic exercise seem to play a larger role than intensity in improving joint health (Brosseau et al., 2017). As such, it is plausible that a longer knitting program (i.e., $30 \mathrm{~min} / \mathrm{session}$ ) might have yielded more positive results.

\subsection{Limitation and future directions}

Several confounding variables could have influenced stiffness and pain, and other outcome measures. Such variables include, but are not limited to, environmental factors (e.g., seasonal changes) and personal factors affecting hand dexterity and strength (e.g., age, handedness, working status, knitting experience). A future study would also do well to assess whether novice and experienced knitters show initial differences in symptoms, and whether they respond differently to the intervention.

Other potential criticisms that could be addressed to the present study include issues related to the population (e.g., variability in HOA disease severity), the intervention (e.g., inability to determine the optimal "dosage" of the intervention in terms of intensity, frequency and duration), the comparison group (e.g., beneficial effect of the educational pamphlet which may mask the effects of the knitting intervention), the randomization process (e.g., not stratified by age, which resulted in the intervention group being slightly older than the control group), the outcomes (e.g., mostly self-reported outcomes), and time measures (e.g., study duration limited to 12 weeks). Regarding this last point, it appeared unethical to place a study participant on a long-term waiting list without an active intervention; considering that our control condition consisted in a waitlist, we could not afford to have our study duration be over 12 weeks ( 8 week knitting program plus 4-week follow-up period). A longer treatment period, allowing for participants to become even more proficient and familiar with knitting, might have yielded better results. More importantly, explicitly forbidding our participants to knit during the 4 -week follow-up period meant 
that we could not assess to what extent participants carried on with the activity unprompted (i.e., after active reminders had ceased). Therefore, while we have recorded that participants enjoyed knitting and adhered to the intervention, we have no way of knowing whether this is an activity they would have integrated in their daily life in the long-term.

Finally, the knitting technique used by the participants is another factor that could have affected the outcomes. The distal interphalangeal (DIP), proximal interphalangeal (PIP) and first carpometacarpal (CMC) joints are most commonly affected in HOA (Walker and Helewa, 2004), and pain management for the CMC joint is particularly problematic (Hamasaki et al., 2015). Continental-style knitting, which requires less thumb movements than the English knitting technique, may therefore be a worthwhile alternative for knitters with HOA. In a future study, it might make sense to identify and separate HOA patients based on their main symptomatic digits (thumb vs. fingers). Comparing the efficacy of knitting programs between individuals with less severe and more severe HOA would also be useful to identify HOA individuals who are more likely to respond to this type of intervention.

\section{Conclusion}

Knitting is a feasible, safe, and enjoyable activity for older women with HOA. However, at this time, our results do not suggest that an 8-week knitting program may be beneficial for reducing stiffness and pain, increasing functional status, grip strength and physical activity level, or improving health related QoL and selfefficacy.

\section{Funding}

This pilot RCT was completed with the support of a research grant obtained from the Physiotherapy Foundation of Canada, Women's Health Division Grant for Research in Women's Health and approved by the Board of Governors of the Physiotherapy Foundation of Canada. The funder played no role in the study. Additional support was provided by the University of Ottawa Research Chair (LB) (salary support for research staff) and in kind from Université de Sherbrooke (GL) for some statistical analyses. University of Ottawa's internal funds (NP, PG) covered the costs for local newspaper for participants' recruitment, knitting materials, statistical analyses and study subjects participation compensations. Guillaume Leonard is supported by the Fonds de la recherche en sante du Quebec.

\section{CRediT authorship contribution statement}

Guillaume Leonard: Conceptualization, conceptualized the knitting intervention and designed this pilot RCT, Writing - original draft, established the selection criteria. Nicole Paquet: Conceptualization, conceptualized the knitting intervention and designed this pilot RCT. Paulette Guitard: Conceptualization, conceptualized the knitting intervention and designed this pilot RCT. Karine Toupin-April: Conceptualization, conceptualized the knitting intervention and designed this pilot, established the selection criteria. Sabrina Cavallo: Conceptualization, conceptualized the knitting intervention and designed this pilot RCT. Gail Paterson: Conceptualization, conceptualized the knitting intervention and designed this pilot RCT. Sibel Z. Aydin: established the selection criteria. Alexia Coulombe-Lévêque: Writing - Review \& Editing. Lucie Brosseau: established the selection criteria, Writing - original draft, Conceptualization, conceptualized the knitting intervention and designed this pilot, GAW Conceptualization, was additionally in charge of the data analyses conceptualization for daily data. All authors read and made comments on previous drafts of the manuscript and approved the final manuscript.

\section{Declaration of competing interest}

The authors declare that they have no competing interests.

\section{References}

Aebischer, B., Elsig, S., Taeymans, J., 2016. Effectiveness of physical and occupational therapy on pain, function and quality of life in patients with trapeziometacarpal osteoarthritis - a systematic review and meta-analysis. J. Hand Ther. 21 (1), 5-15.

Allen, K.D., DeVellis, R.F., Renner, J.B., et al., 2007. Validity and factor structure of the AUSCAN Osteoarthritis Hand Index I a community-based sample. Osteoarthritis Cartilage 15 (7), 830-836.

Altman, R., Alarcon, G., Appelrouth, D., et al., 1990. The American College of Rheumatology criteria for the classification and reporting of osteoarthritis of the hand. Arthritis Rheumatol. 33 (11), 1601-1610.

Bellamy, N., Campbell, J., Haraoui, B., et al., 2002a. Dimensionality and clinical importance of pain and disability in hand osteoarthritis: development of the Australian/Canadian (AUSCAN) osteoarthritis hand index. Osteoarthritis Cartilage 10 (11), 855-862.

Bellamy, N., Campbell, J., Haraoui, B., et al., 2002b. Clinimetric properties of the auscan osteoarthritis hand index: an evaluation of reliability, validity and responsiveness. Osteoarthritis Cartilage 10 (11), 863-869.

Bellamy, N., Haraoui, B., Buchbinder, R., et al., 1996. Development of a diseasespecific health status measure for hand osteoarthritis clinical trials: assessment of the symptom dimensionality. Scand. J. Rheumatol. 106, 5, 5.

Bement, M., Weyer, A., Hartley, S., et al., 2009. Fatiguing exercise attenuates paininduced corticomotor excitability. Neurosci. Lett. 452 (2), 209-213.

Bement, M., 2009. Exercise-induced hypoalgesia: an evidencebased review. In: Sluka, K.A. (Ed.), Mechanisms and Management of Pain for the Physical Therapist. Wolters Kluwer Health, Philadelphia, PA, pp. 143-166.

Bertozzi, L., Valdes, K., Vanti, C., et al., 2015. Investigation of the effect of conservative interventions in thumb carpometacarpal osteoarthritis: systematic review and meta-analysis. Disabil. Rehabil. 37 (22), 2025-2043.

Bohannon, R.W., 1997. Reference values for extremity muscle strength obtained by hand-held dynamometry from adults aged 20 to 79 years. Arch. Phys. Med. Rehabil. 78, 26-32.

Brosseau, L., Wells, G., Marchand, S., et al., 2005. Randomized controlled trial on low level laser therapy (LLLT) in the treatment of osteoarthritis (OA) of the hand. Laser Surg. Med. 36 (3), 210-219.

Brosseau, L., Wells, G.A., Brooks, S., et al., 2014. People getting a grip on arthritis II: an innovative strategy to implement clinical practice guidelines for rheumatoid arthritis and osteoarthritis patients through Facebook. Health Educ. J. 73 (1), 109-125.

Brosseau, L., Leonard, G., 2017. May knitting a promising pain self-management strategy for older woman with osteoarthritic hands? J. Clin. Rheumatol. 23 (3), 179-180.

Brosseau, L., Taki, J., Desjardins, B., et al., 2017. The Ottawa panel clinical practice guidelines for mind-body exercise programs in the management of knee osteoarthritis. Clin. Rehabil. 31 (5), 582-595.

Brosseau, L., Thevenot, O., MacKiddie, O., et al., 2018. The Ottawa Panel guidelines on programmes involving therapeutic exercise for the management of hand osteoarthritis. Clin. Rehabil. 32 (11), 1449-1471.

Cho, H.J., Morey, V., Kang, J.Y., et al., 2015. Prevalence and risk factors of spine, shoulder, hand, hip, and knee osteoarthritis in community-dwelling Koreans older than age 65 years. Clin. Orthop. Relat. Res. 473 (10), 3307-3314.

Damush, T.M., Perkins, S.M., Mikesky, A.E., et al., 2005. Motivational factors influencing older adults diagnosed with knee osteoarthritis to join and maintain an exercise program. J. Aging Phys. Activ 13 (1), 45-60.

Davenport, B.J., Jansen, V., Yeandle, N., 2012. Pilot randomized controlled trial comparing specific dynamic stability exercises with general exercises for thumb carpometacarpal joint osteoarthritis. J. Hand Ther. 17 (3), 60-67.

Dziedzic, K., Nicholls, E., Hill, S., et al., 2015. Self-management approaches for osteoarthritis in the hand: a $2 \times 2$ factorial randomised trial. Ann. Rheum. Dis. 74 (1), 108-118.

Galdino, G., Romero, T., da Silva, J.F.P., et al., 2014. Acute resistance exercise induces antinociception by activation of the endocannabinoid system in rats. Anesth. Analg. 119 (3), 702-715.

Grotle, M., Hagen, K.B., Natvig, B., et al., 2008. Prevalence and burden of osteoarthritis: results from a population survey in Norway. J. Rheumatol. 35 (4), $677-684$

Guy, W., 1976. ECDEU Assessment Manual for Psychopharmacology (DHEW Publication No. ADM 76-338). US Government Printing Office, Washington, DC.

Hamasaki, T., Lalonde, L., Harris, P., et al., 2015. Efficacy of treatments and pain management for trapeziometacarpal (thumb base) osteoarthritis: protocol for a systematic review. BMJ Open 13 (5), e008904, 10.

Hayden-Wade, H.A., Coleman, K.J., Sallis, J.F., et al., 2003. Validation of the telephone and in-person interview versions of the 7-day PAR. Med. Sci. Sports Exerc. 35 (5), 801-809. 
Hennig, T., Haehre, L., Hornburg, V.T., et al., 2015. Effect of home-based hand exercises in women with hand osteoarthritis: a randomised controlled trial. Ann. Rheum. Dis. 74 (8), 1501-1508.

Hoeger Bement, M.K., Weyer, A., Hartley, S., et al., 2009. Fatiguing exercise attenuates pain-induced corticomotor excitability. Neurosci. Lett. 452, 209-213.

Hoffman, M.D., Hoffman, D.R., 2008. Exercisers achieve greater acute exerciseinduced mood enhancement than nonexercisers. Arch. Phys. Med. Rehabil. 89 (2), 358-363.

Kallman, D.A., Wigley, F.M., Scott, W.W., et al., 1989. New radiographic grading scales for osteoarthritis of the hand. Arthritis Rheumatol. 32 (12), 1584-1591.

Kjeken, I., Grotle, M., Hagen, K.B., et al., 2015. Development of an evidence-based exercise program for people with hand osteoarthritis. Scand. J. Occup. Ther. 22 (2), 103-116.

Kloppenburg, M., Bøyesen, P., Visser, A.W., et al., 2015. Report from the OMERACT hand osteoarthritis working group: set of core domains and preliminary set of instruments for use in clinical trials and observational studies. J. Rheumatol. 42 (11), 2190-2197.

Koltyn, K.F., Arbogast, R.W., 1998. Perception of pain after resistance exercise. Br. J. Sports Med. 32 (1), 20-24.

Koltyn, K.F., 2002. Exercise-induced hypoalgesia and intensity of exercise. Sports Med. 32 (8), 477-487.

Koltyn, K.F., Umeda, M., 2007. Contralateral attenuation of pain after short-duration submaximal isometric exercise. J. Pain 8 (11), 887-892.

Krauss, I., Katzmarek, U., Rieger, M.A., et al., 2017. Motives for physical exercise participation as a basis for the development of patient-oriented exercise interventions in osteoarthritis: a cross-sectional study. Eur. J. Phys. Rehabil. Med. 53 (4), 590-602.

Lefler, C., Armstrong, J., 2004. Exercise in the treatment of osteoarthritis in the hands of the elderly. Clin. Kinesiol. 58 (2), 13-17.

Liu-Ambrose, T.Y., Ashe, M.C., Marra, C., 2010. The physical and chronic conditions research team. Independent and inverse association of healthcare utilisation with physical activity in older adults with multiple chronic conditions. Br. J. Sports Med. 44 (14), 1024-1028.

Liu, R., Damman, W., Kaptein, A.A., et al., 2015. Coping styles and disability in patients with hand osteoarthritis. Rheumatology 55 (3), 411-418.

Lorig, K., Chastain, R.L., Ung, E., et al., 1989. Development and evaluation of a scale to measure the perceived self-efficacy in people with arthritis. Arthritis Rheumatol. 32 (1), 37-44.

Merritt, M., 2012. Comparison of Hand Therapy to Placebo in the Treatment of Thumb Carpometacarpal Osteoarthritis. Texas Woman's University, ProQuest Dissertations Publishing.

Millan, M.J., 2002. Descending control of pain. Prog. Neurobiol. 66 (6), 355-474.

O'Brien, L., 2012. The evidence on ways to improve patient's adherence in hand therapy. J. Hand Ther. 25, 247-250.

Oldfield, R.C., 1971. The assessment and analysis of handedness: the Edinburgh inventory. Neuropsychologia 9 (1), 97-113.

Orbai, A.M., Halls, S., Hewlett, S., et al., 2015. More than just minutes of stiffness in the morning: report from the OMERACT rheumatoid arthritis flare group stiffness breakout sessions. J. Rheumatol. 42 (11), 2182-2184.

Østerås, N., Risberg, M.A., Kvien, T.K., et al., 2013. Hand, hip and knee osteoarthritis in a Norwegian population-based study - the MUST protocol. BMC Muscoskel. Disord. 14 (1), 201, 201.
Rabin, R., Charro, F.D., 2001. EQ-SD: a measure of health status from the EuroQoL Group. Ann. Med. 33 (5), 337-343.

Ray, C.A., Carter, J.R., 2007. Central modulation of exercise-induced muscle pain in humans. J. Physiol. 585 (1), 287-294.

Sallis, J.F., Buono, M.J., Roby, J.J., et al., 1997. Seven-day physical activity recall. Med. Sci. Sports Exerc. 29, 89-103.

Sallis, J.F., Haskell, W.L., Wood, P.D., et al., 1985. Physical activity assessment methodology in the Five-City Project. Am. J. Epidemiol. 121 (1), 91-106.

Schreuders, T.A., Roebroeck, M.E., Goumans, J., van Nieuwenhuijzen, J.F., Stijnen, T.H., Stam, H.J., 2003. Measurement error in grip and pinch force measurements in patients with hand injuries. Phys. Ther. 83, 806-815.

Schutzer, K.A., Graves, B.S., 2004. Barriers and motivations to exercise in older adults. Prev. Med. 39 (5), 1056-1061.

Scott, J., Huskisson, E.C., 1976. Graphic representation of pain. Pain 2 (2), 175-184.

Shankland, B., Beaton, D., Ahmed, S., et al., 2017. Effects of client centered multimodal treatment on impairment, function, and satisfaction of people with thumb carpometacarpal osteoarthritis. J. Hand Ther. 30, 303-313.

Slade, S.C., Dionne, C.E., Underwood, M., et al., 2016. Consensus on exercise reporting template (CERT): a modified delphi study. Phys. Ther. 96, 1514-1524.

The Arthritis Society (TAS), 2009. Osteoarthritis: Causes, Symptoms and Treatments. The Conditions Series. http://www.arthritis.ca/.

Polatajko, H., Davis, J., Stewart, D., Cantin, N., Amoroso, B., Purdie, L. Zimmerman, D., 2007. Specifying the domain of concern: Ocupation as core. In: Townsend, E.A., Polatajko, H.J. (Eds.), Enabling occupation II: Advancing an occupational therapy vision for health, well-being, and justice through occupation. CAOT Publications ACE, Ottawa, ON.

Valdes, K., Marik, T., 2010. A systematic review of conservative interventions for osteoarthritis of the hand. J. Hand Ther. 23 (4), 334-351.

Van Tulder, M.W., Assendett, W.J., Koes, B.W., et al., 1997. Editorial Board of the Cochrane Collaboration Back Review Group. Method guidelines for systematic reviews in the Cochrane collaboration back review group for spinal disorders. Spine 22, 2323-2330.

Veale, J.F., 2014. Edinburgh Handedness Inventory - short form: a revised version based on confirmatory factor analysis. Laterality 19 (2), 164-177.

Villafañe, J.H., Cleland, J.A., Fernandez-De-Las-Penas, C., 2013. The effectiveness of a manual therapy and exercise protocol in patients with thumb carpometacarpal osteoarthritis: a randomized controlled trial. J. Orthop. Sports Phys. Ther. 43 (4), 204-213.

Villafañe, J.H., Valdes, K., Vanti, C., et al., 2015. Reliability of handgrip strength test in elderly subjects with unilateral thumb carpometacarpal osteoarthritis. Hand (NY) 10 (2), 205-209.

Vlieland, T.P.V., Zwinderman, A.H., Breedveld, F.C., et al., 1997. Measurement of morning stiffness in rheumatoid arthritis clinical trials. J. Clin. Epidemiol. 50 (7), 757-763.

Walker, J.M., Helewa, A., 2004. Physical Therapy in Arthritis. W.B. Saunders Co, Philadelphia.

Wampold, B.E., 2001. Contextualizing psychotherapy as a healing practice: culture, history, and methods. Appl. Prev. Psychol. 10 (2), 69-86.

Ye, L., Kalichman, L., Spittle, A., et al., 2011. Effects of rehabilitative interventions on pain, function and physical impairments in people with hand osteoarthritis: a systematic review. Arthritis Res. Ther. 13 (1), R28. 